\title{
A Unified Approach to Transient Stability Contingency Filtering, Ranking and Assessment
}

\author{
Damien Ernst, Student Member, IEEE, Daniel Ruiz-Vega, Student Member, IEEE, Mania Pavella, Fellow, IEEE, \\ Peter M. Hirsch, Senior Member, IEEE, and Dejan Sobajic, Senior Member, IEEE
}

\begin{abstract}
This paper proposes a unified approach to contingency filtering, ranking and assessment in power system transient stability studies. The approach consists of two-block techniques in which the first block selects from a list of contingencies the a priori "interesting" ones, that the second block ranks and assesses. This Filtering, Ranking and Assessment (FILTRA) approach relies on SIME (for SIngle Machine Equivalent). SIME is a hybrid direct-time-domain stability method which combines accuracy and flexibility skills of time-domain methods with sound additional possibilities of direct methods. The FILTRA approach is fully general: it may adapt itself to the specifics of any power system (modeling, protection characteristics, etc.), any contingency scenario and mode of (in)stability (first- or multi-swing, local or inter-area mode) and any application context (planning, operation planning and real-time operation). The approach is illustrated on two EHV power systems, having different structures, control and protective devices. The adequacy of its filtering and ranking capabilities is illustrated in terms of performance criteria such as reliability (ability to consistently capture all dangerous contingencies), effectiveness (ability to avoid false alarms) and computational efficiency (ability to comply with real-time requirements). Further, the assessment task is shown to provide the operator with sound information and effective means of control.
\end{abstract}

Index Terms-Contingency filtering and ranking, dynamic security assessment, power system transient stability assessment and control, SIME method.

\section{INTRODUCTION}

I $\mathrm{N}$ TRANSIENT stability studies, contingency filtering and ranking are important but challenging tasks. This holds true for all application contexts, and especially for real-time operation.

Time-domain methods can hardly tackle such tasks for want of adequate stability margins. They can certainly compute stability limits (critical clearing times or power limits); but they would require prohibitive computing times to handle a list of, say, some tens of contingencies. These methods could also classify contingencies into "stable" and "unstable" with respect to a given clearing time, but in a rather crude and inefficient way; indeed, in this case, they would be unable to rank the "interesting"

Manuscript received November 30, 1999. This work was supported by the Electric Power Research Institute (project WO\#3103-10).

D. Ernst is with the University of Liège, Department of Electrical Engineering, Sart-Tilman B28, B-4000 Liège, Belgium. He is also with Research Associate, FNRS.

D. Ruiz-Vega and M. Pavella are with the University of Liège, Department of Electrical Engineering, Sart-Tilman B28, B-4000 Liège, Belgium.

P. M. Hirsch and D. Sobajic are with the Electric Power Research Institute, Palo Alto, CA, USA.

Publisher Item Identifier S 0885-8950(01)06065-5. (i.e., the unstable) contingencies, while, in addition, they would spend considerable amount of CPU time to identify the stable, i.e., the "uninteresting" ones.

Direct or hybrid direct-time-domain methods are intrinsically better suited for such tasks. This paper relies on the hybrid method called SIME (for SIngle Machine Equivalent) to devise a technique which, besides filtering and ranking contingencies, assesses the "interesting" ones in a very informative way.

Basically, the SIME method drives a time-domain program in order to transform the trajectories of a multi-machine system into the trajectory of a One-Machine Infinite Bus (OMIB) equivalent. ${ }^{1}$ A detailed description of SIME may be found in earlier publications (e.g., see [1], [2]), whereas Appendix A glances at its essentials. Let us only mention at once two noteworthy properties. i) By refreshing the OMIB parameters at each step of the time-domain program that it drives, SIME achieves an as accurate stability assessment as this program. ii) SIME does not intend to replace this program but, rather, to complement it with multiform information provided by the combination of the OMIB and the equal-area criterion; in particular, with stability margins and critical machines, which are the core of the proposed approach.

This "filtering-ranking-assessment" (FILTRA) approach consists of two successive blocks: one for filtering and one for ranking and assessing contingencies. As will appear in the following sections, this structure yields a unified approach in many respects: i) the same transient stability package is used to filter, rank and assess contingencies; ii) the information obtained in the first block is also used in the second block; iii) the information obtained in the second block is used in various ways for ranking contingencies, assessing them and finally stabilizing them. Another interesting feature is the great flexibility in the design of the two blocks. In particular, the filtering block may comprise more than one sub-block, using increasing modeling sophistication so as to discard at the early stages the most stable contingencies (see Appendix B). Yet another asset of the approach is its ability to capture all dangerous contingencies, and to avoid false alarms to a great extent.

The FILTRA procedure is elaborated in Section II and scrutinized in Section III via simulations performed on two different power systems: the EPRI test system C [5], and the HydroQuébec system. It is shown that the technique is consistently reliable (i.e., able to capture all the dangerous contingencies),

\footnotetext{
${ }^{1}$ This OMIB transformation generalizes the one used in the Extended Equal Area Criterion (EEAC) method [3]. In this respect, SIME may be considered as a generalization of the EEAC [4].
} 

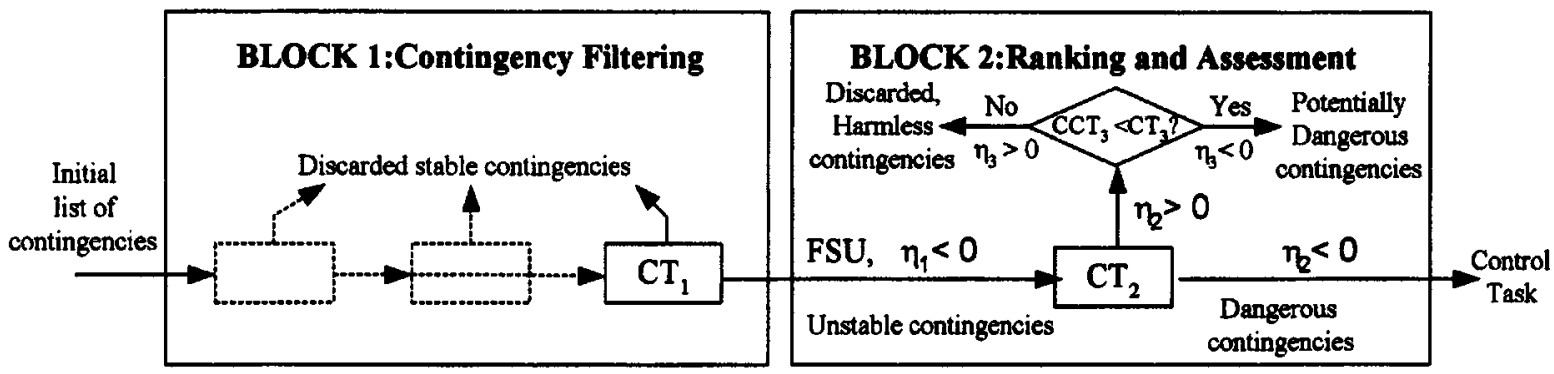

Fig. 1. Principle of the proposed FILTRA technique.

that ranks correctly the "interesting" ones, and finally that assesses these latter in a way meaningful to the system operator. Further, it is shown that these various tasks are achieved within computing times compatible with on-line requirements. Finally, control issues are addressed, based on information provided by the FILTRA approach.

N.B. Giving credit to the large number of nonconventional contingency filtering approaches proposed in the technical literature would be hardly possible. The interested reader is kindly referred to surveys or dedicated monographs [6]-[9].

\section{Filtering-RANKING-ASSESSMENT (FILTRA) APPROACH}

The proposed approach is designed so as to meet key requirements stated in Section II-A. The resulting general structure is elaborated in Section II-B and portrayed in Fig. 1. Its mechanism is scrutinized and main properties are highlighted in Section II-C.

\section{A. Problem Statement}

Any good contingency filter should meet some key requirements, expressed hereafter in terms of conditions. Main terms used in the remainder of the paper are also defined.

Condition 1: Classification ability. A good classifier should be able to screen and rank contingencies on the basis of increasingly severe criteria. In the FILTRA approach, the various contingencies are classified into first-swing stable or unstable with respect to a long clearing time, $\mathrm{CT}_{1}$. These latter are then classified into (multi-swing) harmless $(\mathbf{H})$, potentially dangerous or dangerous with respect to a second clearing time, $\mathrm{CT}_{2}$, shorter than $\mathrm{CT}_{1}$. Further, the dangerous contingencies are ranked according to their degree of severity and assessed. These terms are defined below and illustrated in Fig. 1. A contingency is said to be

- Dangerous (D) if its occurrence drives the system out of step; in other words, a contingency whose critical clearing time is smaller than the time response of system protections;

- Potentially Dangerous (PD) if it is "almost" dangerous, i.e., milder than, but likely to become dangerous under slightly modified operating conditions;

- First-Swing Unstable (FSU) [respectively Stable (FSS)], if under given clearing scenario it drives the system to first-swing instability (respectively stability).

The severity criterion used in the above definitions is contingency clearing time (CT). To classify a contingency as FSS or FSU, the filtering block chooses a first threshold, $\mathrm{CT}_{1}$, quite

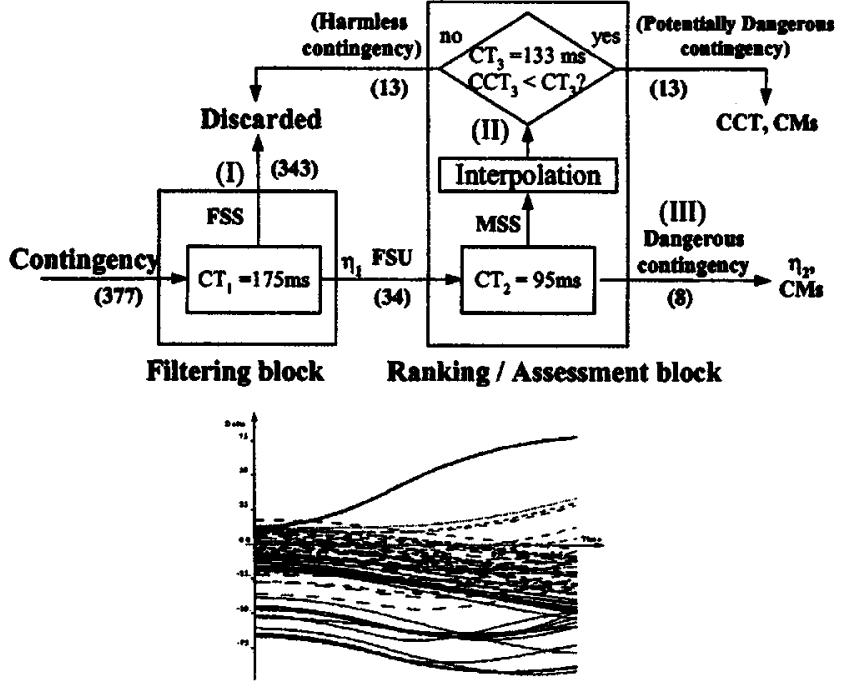

(I)

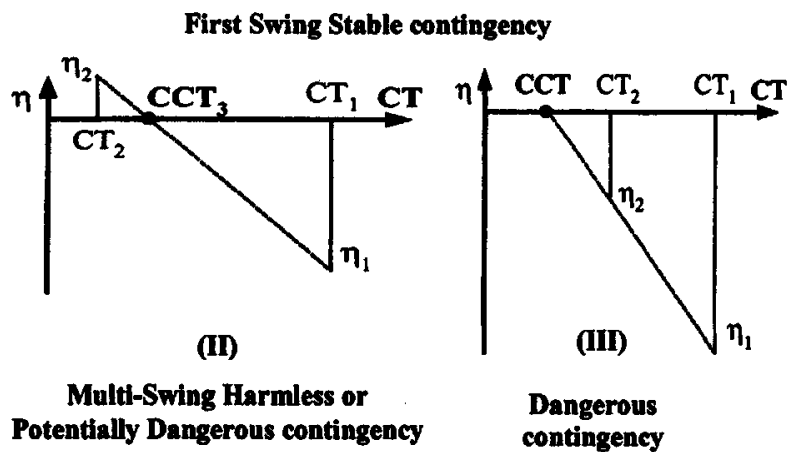

Fig. 2. A realization of the FILTRA technique. Schematic description of the various contingency classes.

larger than the time response of the system protections of concern. Further, to rank an FSU contingency, the second block chooses a second threshold, $\mathrm{CT}_{2}$, slightly larger than the protections' time response: accordingly, it declares an FSU contingency to be $\mathrm{D}$, if it is unstable for this $\mathrm{CT}_{2}$; otherwise, it ranks it as $\mathrm{H}$ or PD depending upon whether it is stable or unstable with respect to a third threshold value, $\mathrm{CT}_{3}$ (see Fig. 2).

Condition 2: Accuracy. The unstable contingencies must be assessed accurately. This is achieved by using in block 2 detailed power system models to rank and assess the first-swing unstable contingencies injected to this block.

Condition 3: Reliability. The contingency filter must be extremely reliable, i.e., able to capture all the dangerous contingencies. This is achieved by the combined use of detailed system 
models and fairly large threshold values for $\mathrm{CT}_{1}$ (see also below, Section II-B).

Condition 4: Efficacy. The contingency filter should have an as low as possible rate of false alarms, i.e., of contingencies suspected to be dangerous while they are not.

Note that the identification of all dangerous contingencies is a condition of paramount importance for the very validity of the filter, while false alarms may impact on its computing skills.

Condition 5: Computational efficiency. The overall procedure of contingency filtering, ranking and assessment should be as fast as possible, whatever the application context. This condition becomes crucial when it comes to real-time operation. The computing requirements of the FILTRA approach are assessed in Section II-C-5.

Remark: A handy measure for assessing computational performances: sTDI. To assess computational performances of the FILTRA technique, first note that the computing effort required by SIME itself is virtually negligible with respect to any other task. (To fix ideas, it corresponds to less than one iteration of the power flow program.) Hence, the overall computing effort reduces to that for running the time-domain program during the short periods required by SIME. Therefore, a handy means for comparisons appears to be the corresponding seconds of Time-Domain Integration (sTDI for short). Indeed, this "measure" renders comparisons independent of the computer in use and of the system size; note, however, that for a same timedomain program it may correspond to different CPU times, depending upon the simulation range.

\section{B. General Design}

Fig. 1 portrays the general two-block structure of the FILTRA technique. The first block is devoted to the filtering task; it may be made up of several successive sub-blocks, with increasing modeling details and filtering accuracy, as discussed in Appendix B. The second block ranks and assesses the "interesting" contingencies sent from the first block.

As suggested by Fig. 1, contingency filtering and ranking rely on margins $(\eta \mathrm{s})$ computed for two clearing times (CTs), fixed so as to comply with the conditions of Section II-A. Note that $\mathrm{CCT}_{3}$, shown in the upper part of block 2 , is obtained by linear interpolation of $\eta_{1}$, and $\eta_{2}$ (see Section II-C and sketch (II) of Fig. 2). Recall also that $\mathrm{CT}_{3}$ is an intermediate value between $\mathrm{CT}_{1}$ and $\mathrm{CT}_{2}\left(\mathrm{CT}_{1}>\mathrm{CT}_{3}>\mathrm{CT}_{2}\right){ }^{2}$ Margins and $\mathrm{CT}$ s are used as follows.

First Block: Contingency Filtering: Whatever the internal structure, the last step of this block performs a stability computation with detailed power system modeling and contingency clearing time $\mathrm{CT}_{1}$ to classify each contingency as first-swing stable or unstable and, accordingly, to:

- discard the contingency if it is FSS

- send the contingency to block 2 along with its (negative) margin $\eta_{1}$, if it is FSU.

Second Block: Contingency Ranking and Assessment: This block uses detailed power system modeling and a threshold $\mathrm{CT}_{2}$ to compute a stability margin, $\eta_{2}$, and classify a contingency as:

- Dangerous (D), if, $\eta_{2}<0$

${ }^{2}$ See for example the values used in Fig. 2
- Potentially Dangerous (PD) or Harmless (H), if $\eta_{2}>0$ : the $\mathrm{CCT}_{3}$ value resulting from the linear interpolation of $\eta_{1}(<0)$ and $\eta_{2}(>0)$ decides whether the contingency is unstable for $\mathrm{CT}_{3}\left(\mathrm{CCT}_{3}<\mathrm{CT}_{3}\right)$, and hence $\mathrm{PD}$, or stable $\left(\mathrm{CCT}_{3}>\mathrm{CT}_{3}\right)$ and hence $\mathrm{H}$.

To summarize, only the dangerous contingencies would actually threaten the power system, and deserve finer exploration (see Section II-C-3). The potentially dangerous contingencies might be put in a stand-by list and checked after stabilization of the dangerous contingencies.

\section{Remarks:}

1) The reason for using detailed power system modeling in the last step of block 1 is twofold: for better accuracy, and for allowing (inter-) extrapolating the resulting margin with that computed for $\mathrm{CT}_{2}$, in block 2 .

2) Many variants of the FILTRA technique may be thought of in order to comply with power system specifics. They all differ in the structure of the filtering block (see Appendix B), whereas the second block, which carries the main properties of the approach, is less liable to changes.

\section{Illustration on a Particular Structure}

The general FILTRA approach proposed in Section II-B is here scrutinized on the simple structure of Fig. 2, in order to describe its mechanism and uncover main features and properties. This structure will subsequently be used in the simulations of Section III; the parameter values and contingency numbers displayed in this figure are borrowed from these simulations.

1) Contingency Filtering: According to Fig. 2, 377 contingencies are inputted to block 1 . In order to classify them as first-swing stable or unstable, SIME drives the time-domain program, first in the during-fault then in the post-fault configuration entering at $\mathrm{CT}_{1}=175 \mathrm{~ms}$. Further, SIME stops the timedomain integration as soon as one of the three conditions is met: the system extreme machines reach a maximum angular deviation; the OMIB angle reaches a maximum value; the OMIB reaches its unstable conditions (A.3) of Appendix A.

In the first two cases the contingency is declared to be firstswing stable (FSS) and discarded. In the latter case, the contingency is declared first-swing unstable (FSU) and sent to block 2 along with its corresponding negative margin, $\eta_{1}$, and list of critical machines, determined according to Appendix A. For example, in the case of Fig. 2, out of the 377 contingencies, 343 are discarded and 34 are sent to the second block.

2) Contingency Ranking: Following the general pattern of Section II-B, SIME ranks the FSU contingencies by driving the time-domain program with $\mathrm{CT}_{2}=95 \mathrm{~ms}$ onwards. The simulation is either stopped as soon as the instability conditions (A.3) in Appendix A are met or pursued on the entire integration pe$\operatorname{riod}(5,10$ or $15 \mathrm{~s}$, as appropriate), if the simulation is found to be stable. ${ }^{3}$ In the former case, the contingency is declared to be dangerous and the corresponding (negative) margin $\eta_{2}$ is computed; in the latter case, the (positive) margin $\eta_{2}$ is computed and interpolated with $\eta_{1}$ to get $\mathrm{CCT}_{3}$ and: if $\mathrm{CCT}_{3}$ is larger than

\footnotetext{
${ }^{3}$ Note that the reason for performing the time-domain simulation on the entire integration period is to guarantee that the contingency is indeed multi-swing stable; otherwise, i.e., in case of multi-swing instabilities, the contingency is dangerous and treated as such.
} 
$\mathrm{CT}_{3}$, the contingency is harmless and discarded; otherwise, the contingency is potentially dangerous and stored in the "waiting list."

3) Refined Ranking of Dangerous Contingencies: To rank the dangerous contingencies, two parameters obtained as by-products of the above simulations may a priori be considered: the unstable (negative) margin; the time to instability (i.e., the time for the OMIB to reach instability), $t_{u}$.

Concerning margins, observe that the approximate CCT obtained by extrapolating the negative margins, $\eta_{1}$, and $\eta_{2}$ [see sketch (III) of Fig. 2] would be a good "measure" of contingency severity. However, these two margins (especially margin $\eta_{1}$ ) seldom exist for very unstable scenarios like those of dangerous contingencies (see the examples and relating explanations in Section III). Finally, note that the "normalized" margin (margin divided by the OMIB inertia coefficient) would be more suitable than expression (A.1) of Appendix A, since different margins generally correspond to different critical machines (CMs). But, again, this margin does not exist always.

The time to instability, $t_{u}$, seems to be more convenient for ranking contingencies; indeed normally, the more unstable a contingency the faster the system loses synchronism. Observe, however, that only $t_{u}$ 's referring to the same type of instability may be compared. (See a counter-example and its discussion in Section III). Note that Ref. [10] uses also the time to instability, though computed in a different way.

4) Assessment of Dangerous Contingencies: What mainly characterizes a dangerous contingency is its margin $\eta_{2}$, and corresponding critical machines (CMs). Knowledge of these two pieces of information opens avenues toward control, i.e., stabilization; this may be achieved by assessing how much of the CMs generation should be reported on noncritical machines in order to reach the stability-instability border, i.e., to cancel out $\eta_{2}$. Note that control goes beyond the scope of this paper; it is however shortly treated in Section III-D, to show that it is a straightforward extension of the above assessment.

5) Computing Requirements of the FILTRA Technique: In terms of sTDI's (see Remark of Section II-A), the computing times required to classify contingencies into the above four classes are as follows.

$$
\begin{array}{ll}
\text { FSS: } t_{\mathrm{r}}\left(\mathrm{CT}_{1}\right) ; & \mathrm{D}: t_{\mathbf{u}}\left(\mathrm{CT}_{1}\right)+t_{\mathbf{u}}\left(\mathrm{CT}_{2}\right) \\
\text { PD: } t_{\mathbf{u}}\left(\mathrm{CT}_{1}\right)+M I P ; & \mathrm{H}: t_{\mathbf{u}}\left(\mathrm{CT}_{1}\right)+M I P .
\end{array}
$$

In the above, $t_{\mathrm{r}}\left(\mathrm{CT}_{1}\right)$ denotes the time to reach the first-swing stable conditions. Similarly, $t_{\mathbf{u}}\left(\mathrm{CT}_{1}\right)$ [respectively $t_{\mathbf{u}}\left(\mathrm{CT}_{2}\right)$ ] is the time to reach the unstable conditions for $\mathrm{CT}=\mathrm{CT}_{1}$ (respectively $\mathrm{CT}_{2}$ ). Finally, $M I P$ denotes the "Maximum Integration Period" (e.g., in Section III it is taken equal to $5 \mathrm{~s}$ for the EPRI system and $10 \mathrm{~s}$ for the Hydro-Québec system).

Note that the refined ranking proposed in Section II-C-3 does not require any additional computing time.

6) Main Properties of the FILTRA Approach: Let us summarize the properties uncovered so far.

1) The approach appears to be truly "unified" and straightforward. Indeed, it uses the same SIME program throughout. Further, the resulting pieces of information are generally used twice: thus, the margin $\eta_{1}$, computed
TABLE I

\begin{tabular}{|c|c|c|c|c|c|c|}
\hline 1 & 2 & 3 & 4 & 5 & 6 & 7 \\
\hline \multirow[t]{2}{*}{$\begin{array}{l}\text { Power } \\
\text { system }\end{array}$} & \multirow[t]{2}{*}{$\begin{array}{l}\text { Nr of } \\
\text { Buses }\end{array}$} & \multirow[t]{2}{*}{$\begin{array}{l}\text { Nr of } \\
\text { lines }\end{array}$} & \multicolumn{2}{|c|}{$\begin{array}{l}\text { Machine } \\
\text { Modelling }\end{array}$} & \multirow{2}{*}{$\begin{array}{l}\text { Total } \\
\text { Power } \\
\text { (MW) }\end{array}$} & \multirow{2}{*}{$\begin{array}{c}\mathrm{Nr} \\
\text { of } \\
\text { cont }\end{array}$} \\
\hline & & & DM & SM & & \\
\hline EPRI & 434 & 2357 & 14 & 74 & 350,749 & 252 \\
\hline $\begin{array}{l}\text { Hydro- } \\
\text { Québec }\end{array}$ & 661 & 858 & 94 & 0 & 36,682 & 377 \\
\hline
\end{tabular}

POWER SYSTEMS MAIN CHARACTERISTICS

at the filtering block to screen FSS contingencies is subsequently used in the second block, together with the margin $\eta_{2}$ to rank the FSU contingencies; similarly, the margin $\eta_{2}$ is subsequently used to assess the severity of the D contingencies and, if desired, of the PD contingencies as well.

2) The very stable cases are assessed only approximately, thus requiring little CPU time.

3) The more unstable a contingency, and the more detailed and accurate the information provided about it.

4) The procedure is extremely reliable, in that it is designed so as to capture all dangerous contingencies; this reliability is obtained at the expense of a low rate of false alarms.

5) The above properties contribute to make the procedure computationally very efficient and compatible with on-line requirements. Besides, the most time-consuming steps may easily be parallelized and performed by distributed computing.

\section{SimulationS}

\section{A. Simulation Description}

Two power systems are considered: the EPRI test C [5] and the Hydro-Québec (H-Q) power system. Their main characteristics are summarized in Table I. Columns 4 and 5 of the table indicate the number of machines with detailed model (DM) and with simplified model (SM) respectively. On both systems, the contingencies considered are 3- $\phi$ short-circuits, applied at EHV buses $(500 \mathrm{kV}$ for the EPRI system and 735, 345 and $315 \mathrm{kV}$ for the H-Q system); they are cleared by tripping one or several lines. Note that the 252 contingencies mentioned in the table for the EPRI system result from the simulation of 36 contingencies under 7 different operating conditions.

For both power systems, the FILTRA structure and parameters are those displayed in Fig. 2. The number of contingencies displayed in the figure and the results correspond to the simulations of the H-Q system. The time-domain program used for the EPRI system is ETMSP [11], and for the H-Q system is ST-600 [12]. These programs are coupled with SIME for the needs of the FILTRA technique, and also as reference for accuracy comparisons. Note that in order to comply with operational uses, the maximum integration period for a stable simulation was fixed at $5 \mathrm{~s}$ for the EPRI system and $10 \mathrm{~s}$ for the H-Q system.

\section{B. Simulation Results}

1) Filtering Block: For the EPRI system, out of the initial list of 252 contingencies, 172 have been found FSS and 
TABLE II

RANKING AND ASSESSMENT OF DANGEROUS CONTINGENCIES

\begin{tabular}{|c|c|c|c|c|c|c|}
\hline 1 & 2 & 3 & 4 & 5 & 6 & 7 \\
\hline $\begin{array}{c}\text { Cont. } \\
\mathrm{Nr}\end{array}$ & $\begin{array}{c}\eta_{2} \\
(\mathrm{rad} / \mathrm{s})^{2}\end{array}$ & $\begin{array}{c}\mathrm{Nr} \\
\text { of } \\
\mathrm{CMs}\end{array}$ & $\begin{array}{c}P c \\
(\mathbf{M} W)\end{array}$ & $\begin{array}{c}\left(t_{\mathrm{u}}\right) t_{\mathrm{u} 2} \\
(\mathrm{~ms})\end{array}$ & $\mathbf{R}$ & $\begin{array}{l}\text { CCT } \\
\text { (ms) }\end{array}$ \\
\hline \multicolumn{7}{|c|}{ EPRI test system } \\
\hline 1 & * & 6 & 4,832 & $(485) 395$ & 1 & 0 \\
\hline 30 & -1.20 & 28 & 20,008 & (550) 1010 & 2 & 0 \\
\hline 11 & -0.81 & 37 & 26,938 & (365) 1325 & 3 & 66 \\
\hline 10 & -0.70 & 39 & 27,714 & (390) 1395 & 4 & 70 \\
\hline \multicolumn{7}{|c|}{ Hydro-Québec system } \\
\hline 33 & * & 5 & 5572 & $(258) 320$ & 1 & 0 \\
\hline 39 & * & 5 & 5572 & (258) 322 & 2 & 0 \\
\hline 1405 & * & 5 & 5572 & (284) 364 & 3 & 0 \\
\hline 13 & * & 5 & 5572 & (284) 364 & 4 & 0 \\
\hline 1415 & -5.41 & 5 & 5572 & (332) 408 & 5 & 48 \\
\hline 243 & -0.85 & 8 & 2293 & (324) $971^{*}$ & 6 & 67 \\
\hline 37 & -4.73 & 5 & 5572 & (260) 414 & 7 & 80 \\
\hline 636 & -1.74 & 1 & 42 & (258) 921 & 8 & 92 \\
\hline
\end{tabular}

discarded. The remaining 80 FSU contingencies have been selected and sent to the second block for ranking and assessment.

For the H-Q system, out of the initial list of 377 contingencies, 343 were found to be FSS and 34 FSU.

2) Ranking and Assessment Block: For the EPRI system: the 80 FSU contingencies are decomposed into $31 \mathrm{H}, 25 \mathrm{PD}$ and $24 \mathrm{D}$ contingencies. For the H-Q system: the $34 \mathrm{FSU}$ contingencies are decomposed into $13 \mathrm{H}, 13 \mathrm{PD}$ and $8 \mathrm{D}$ contingencies.

The dangerous contingencies are further ranked, according to Section II-C-3. The obtained results are gathered in Table II, where:

column 2 gives the margin $\eta_{2}$ computed for $\mathrm{CT}=$ $95 \mathrm{~ms}$. An asterisk indicates that there is no margin; this happens when the $\mathrm{P}_{\mathrm{e}}$ curve does not intersect the $P_{m}$ curve, i.e., when $P_{a}$ remains always negative in the $P-\delta$ representation of Fig. 3(a): there is no equilibrium solution in the post-fault system. Obviously such cases are very unstable;

- column 3 specifies the number of critical machines;

- column 4 lists the total power generated by these CMs. This information is quite useful, though not crucial;

- column 5 gives the time to instability, defined in Section II-C-3: the first, between brackets, refers to the first simulation, using $\mathrm{CT}_{1}=175 \mathrm{~ms}$; the second to the second simulation, using $\mathrm{CT}_{2}=95 \mathrm{~ms}$. Note that all the dangerous contingencies are first-swing unstable, apart from contingency $\mathrm{Nr} 243$ of the H-Q system which loses synchronism after a back-swing excursion;

- column 6 ranks the contingencies in increasing order of $t_{\mathbf{u} 2}$ (apart from contingency 243, which has a different mode of instability).

- column 7 provides the reference CCTs furnished by the full SIME, i.e., the SIME program run with decreasing clearing times, the last simulation being run on the entire integration period.

Finally, we mention that for the EPRI system only the 4 dangerous contingencies corresponding to operating point $\mathrm{Nr} 6$ [5], [11] are displayed, the others exhibiting quite similar behavior.

\section{3) Comments:}

1) Concerning contingency ranking, observe that use of margins is not convenient; in particular, because most of the dangerous contingencies do not have margin for $\mathrm{CT}_{2}=$ $95 \mathrm{~ms}$, and a fortiori for $\mathrm{CT}_{1}=175 \mathrm{~ms}$.

On the contrary, time to instability seems to be a convenient contingency severity indicator: the ranking of column 6 of Table II coincides with that relying on the reference CCTs of last column, except for contingency $\mathrm{Nr} 243$.

2) The distribution of contingencies into FSS, H, PD and D is much more realistic for the H-Q than for the EPRI system, where the 252 contingencies under consideration seem to result from a pre-selection having discarded most of the stable contingencies.

4) Illustrating the Behavior of Various Types of Contingencies: Appendix A uses three different representations to describe contingency behavior, namely: multimachine swing curves, OMIB swing curves and OMIB $P-\delta$ curves. More precisely, Fig. 1(a) deals with a harmless contingency (contingency $\mathrm{Nr} 3$ of EPRI test system C), simulated under two clearing times: $\mathrm{CT}_{1}=175 \mathrm{~ms}$ (for which the contingency is FSU, $\eta_{1}<0$ ) and $\mathrm{CT}_{2}=95 \mathrm{~ms}$ (the contingency is stable, $\left.\eta_{2}>0\right)$ ). We mention that interpolation of these two margins provides an approximate CCT of $153 \mathrm{~ms}$, while SIME and ETMSP programs furnish 161 and $156 \mathrm{~ms}$ respectively. Hence, the contingency is classified as harmless $\left(\mathrm{CCT}_{3}=153 \mathrm{~ms}>\right.$ $\mathrm{CT}_{3}=133 \mathrm{~ms}$ ). Note that a potentially dangerous contingency exhibits similar behavior and representations (by definition it is unstable for $\mathrm{CT}_{1}$ and stable for $\mathrm{CT}_{2}$ but, in this case, $\mathrm{CCT}_{3}$ $<\mathrm{CT}_{3}$ ).

On the other hand, Fig. 4 is relative to the dangerous contingency Nr. 10 of EPRI test system C. We mention that this contingency is first-swing unstable for $\mathrm{CT}_{2}=95 \mathrm{~ms}$ (loss of synchronism at $t_{\mathbf{u}}=1.325 \mathrm{~s}$ ). To illustrate multiswing instabilities, a shorter clearing time ( $20 \mathrm{~ms}$ instead of $95 \mathrm{~ms})$ and larger maximum integration period ( $15 \mathrm{~s}$ instead of $5 \mathrm{~s})$ were considered. The system is multiswing unstable for this new CT $=20 \mathrm{~ms}$ losing synchronism at $t_{\mathbf{u}}=13.7 \mathrm{~s}$. Observe that the OMIB swing curve allows a clearer description of the multiswing phenomena than the multimachine swing curves.

\section{Performances}

1) Reliability: Simulations not reported here show that all contingencies discarded by the filtering block are indeed stable, and that all dangerous contingencies have properly been captured. Note also that the CCTs obtained with the time-domain programs run alone are found in perfect agreement with the CCTs of the full SIME [13].

2) Computational Efficiency: The only needlessly lengthy computation is the one concerning the harmless contingencies : 13 out of the 377 for the H-Q system, and 13 out of the 252 for the EPRI system. This is worth for guaranteeing full reliability.

3) Ranking Ability of the FILTRA Procedure: very good, according to the comparison of columns 6 and 7 of Table II.

4) Computing Performances: The computing times required by the FILTRA simulations on the H-Q system are 

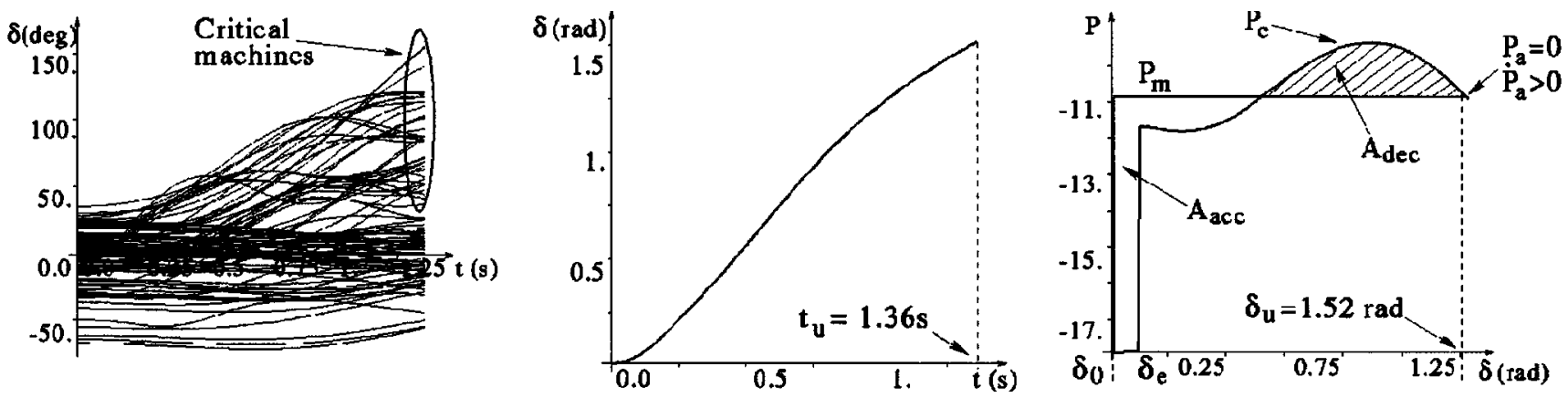

(a) Multimachine swing curves, OMIB swing curve and OMIB P- $\delta$ representation on a unstable simulation: CT $=175 \mathrm{~ms}$
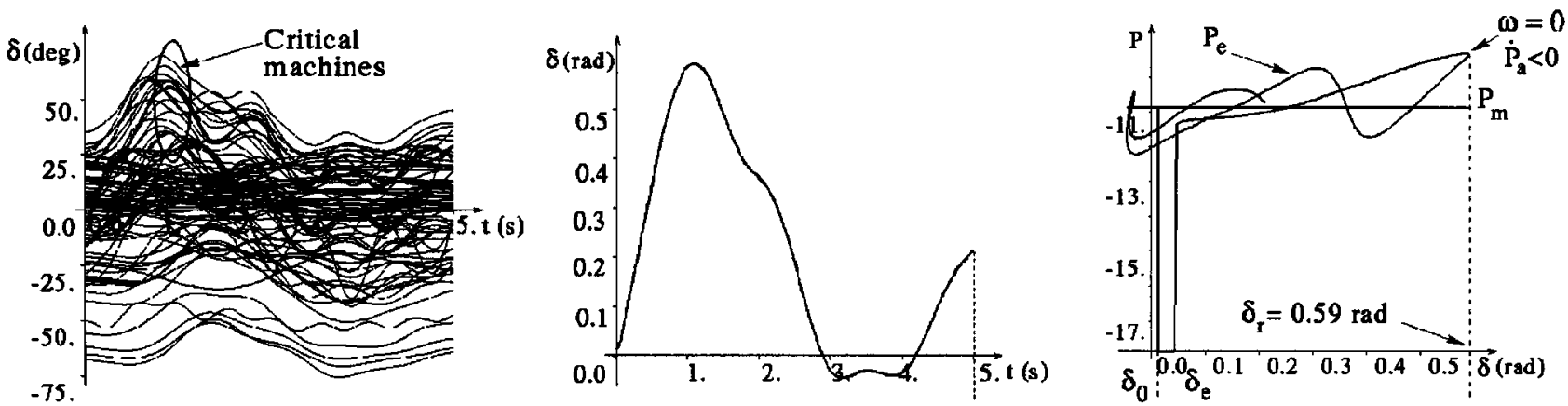

(b) Multimachine swing curves, OMIB swing curve and OMIB P- $\delta$ representation on a stable simulation: $\mathrm{CT}=95 \mathrm{~ms}$

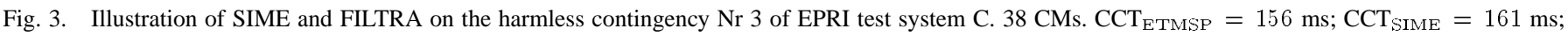
$\mathrm{CCT}_{\text {FILTRA }}=153 \mathrm{~ms}$.
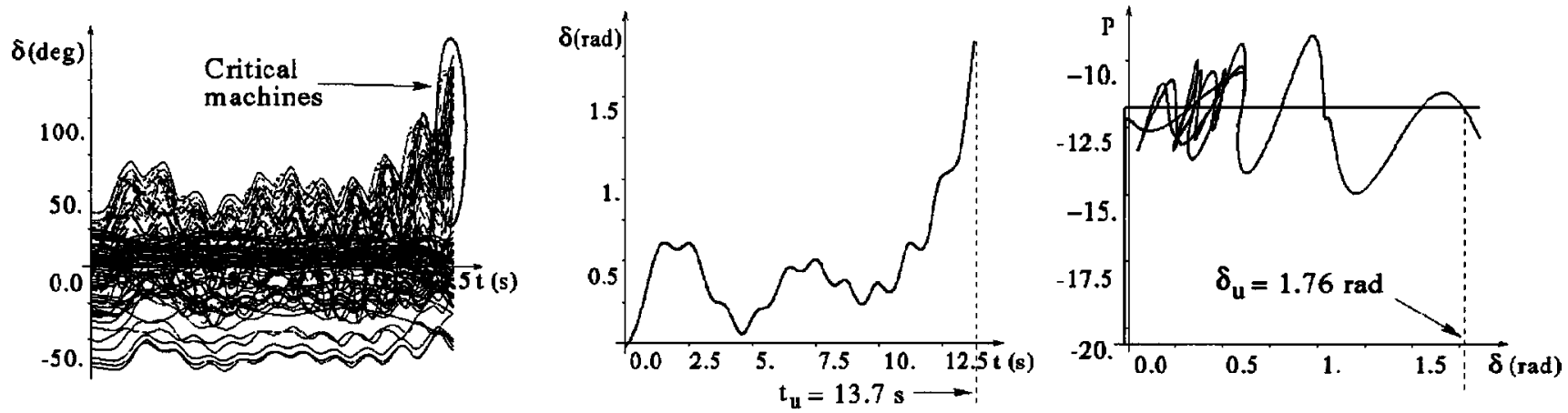

Fig. 4. Multimachine swing curves, OMIB swing curve and OMIB P- $\delta$ representation on a multiswing unstable simulation of contingency Nr. 10 of EPRI test system C with $\mathrm{CT}=20 \mathrm{~ms} .39 \mathrm{CMs}$. Using a maximum integration period of $15 \mathrm{~s} . \mathrm{CCT}_{\mathrm{ETMSP}}=\mathrm{CCT}_{\mathrm{STMF}}=0 \mathrm{~ms}$.

assessed in terms of sTDI. According to the considerations of Section II-C-5, this yields the following global values.

$$
\begin{array}{ll}
\text { FSS: } \operatorname{tr}\left(\mathrm{CT}_{1}\right) & =141.6 \\
\text { D: } \operatorname{tu}\left(\mathrm{CT}_{1}\right)+\operatorname{tu}\left(\mathrm{CT}_{2}\right)=2.3+4.1 & =6.4 \\
\text { PD: } \operatorname{tu}\left(\mathrm{CT}_{1}\right)+\mathrm{MIP}=5.5+130 & =135.5 \\
\text { H: } \operatorname{tu}\left(\mathrm{CT}_{1}\right)+\mathrm{MIP}=5.9+130 & =135.9 \\
\text { Total time: } & 419.4 \text { sTDI. }
\end{array}
$$

This total may be decomposed into the time required by:

- the first block, which amounts to 155.3 sTDI,

- the second block, which amounts to 264.1 sTDI.

Of the above 264.1 sTDI, 260 sTDI are spent to run 26 stable simulations on the entire integration period (10 sTDI per contingency), while the dangerous contingencies require only a few percentage (about 1.5\%). In other words, apart from the contingency filtering of the first block, which is unavoidable, most of the computing time is spent to explore existence of multi-swing phenomena. This computation might be avoided if such phenomena are not of concern (for example, if the system operator knows by experience that they don't exist).

In this latter case, i.e., if multi-swing phenomena are not sought, the time for computing the $\mathrm{D}, \mathrm{PD}$ and the $\mathrm{H}$ contingencies reduces to about $30.5 \mathrm{sTDI}$, and the total computing effort from 419.4 to 172.0 sTDI. They correspond to mean computing times of, respectively, 1.1 and 0.5 sTDI per contingency.

\section{Assessment and Control of Dangerous Contingencies}

The issue of control goes beyond the objective of this paper. It is however interesting to shortly illustrate how the assessment of dangerous contingencies provided by the FILTRA procedure (at the output of the second block of Fig. 2) may be used to stabilize them readily and automatically. 
TABLE III

StABILIZING DANGEROUS CONTINGENCIES

\begin{tabular}{|c|c|c|c|c|c|c|}
\hline 1 & 2 & 3 & 4 & 5 & 6 & 7 \\
\hline $\begin{array}{c}\text { Iter. } \\
\mathrm{Nr}\end{array}$ & $\begin{array}{c}\eta \\
\text { (rad./s) }\end{array}$ & $\begin{array}{l}\mathrm{Nr} \text { of } \\
\mathrm{CMs}\end{array}$ & $\begin{array}{c}\text { Pci } \\
(\mathbf{M W})\end{array}$ & $\begin{array}{c}\Delta \mathbf{P c} \\
(\mathbf{M W})\end{array}$ & $\begin{array}{c}\text { Pcf } \\
(\mathrm{MW})\end{array}$ & $\begin{array}{c}t_{u} \\
(\mathrm{~ms})\end{array}$ \\
\hline \multicolumn{7}{|c|}{ EPRI test system } \\
\hline \multicolumn{7}{|c|}{ Cont. I } \\
\hline 0 & $-12.55^{*}$ & 6 & 4831 & -441 & 4392 & 395 \\
\hline 1 & $-10.30^{*}$ & 7 & 5162 & Extr. & 3448 & 460 \\
\hline 2 & $-1.74^{*}$ & 7 & 3448 & Extr. & 2826 & 745 \\
\hline 3 & -2.83 & 7 & 2826 & -254 & 2572 & 1040 \\
\hline 4 & -0.90 & 7 & 2572 & Extr. & 2454 & 1320 \\
\hline 5 & 0.37 & 7 & 2454 & Inter. & 2489 & 5000 \\
\hline \multicolumn{7}{|c|}{ Cont. 30} \\
\hline 0 & -1.20 & 28 & 20008 & -583 & 19425 & 1010 \\
\hline 1 & -0.31 & 16 & 9175 & -268 & 8907 & 1625 \\
\hline 2 & 0.0 & 39 & 26889 & $\cdots$ & -- & 3025 \\
\hline \multicolumn{7}{|c|}{ Hydro-Québec system } \\
\hline \multicolumn{7}{|c|}{ Cont. 13} \\
\hline 0 & $-2.01 *$ & 5 & 5572 & -507 & 5065 & 364 \\
\hline 1 & -4.53 & 5 & 5065 & -148 & 4917 & 470 \\
\hline 2 & -2.12 & 5 & 4917 & Extr. & 4787 & 670 \\
\hline 3 & 0.07 & 5 & 4787 & Inter. & 4791 & 10000 \\
\hline \multicolumn{7}{|c|}{ Cont. 37} \\
\hline 0 & -4.73 & 5 & 5572 & -163 & 5409 & 414 \\
\hline 1 & -1.97 & 5 & 5409 & Extr. & 5292 & 556 \\
\hline 2 & 1.1 & 5 & 5292 & Inter. & 5334 & 2000 \\
\hline
\end{tabular}

This stabilization relies on the knowledge of the unstable margin $\eta_{2}$ and corresponding CMs, and consists of acting on the power system generation so as to cancel out this margin. In turn, the equal-area criterion (see in Fig. 3) suggests that this may be achieved by adjusting the mechanical power of the OMIB or, equivalently, of the critical machines, Pc; further, in order to meet the load, the power decrease in CMs must be compensated by an (almost) equal increase in noncritical machines.

To assess the amount of Pc decrease necessary to stabilize an unstable scenario, a compensation scheme was proposed in Refs. [14], [15]. In this paragraph, a more pragmatic procedure is used; it consists of decreasing Pc by a factor of 1.03 or 1.1, depending upon whether an initial margin exists or not. Table III summarizes the results obtained with four dangerous contingencies, two for each power system (one with, the other without margin). Column 2 of the table provides the margin values; in their absence, the asterisk indicates that, instead, the "minimum distance" between the $\mathrm{P}_{\mathrm{m}}$ and $\mathrm{P}_{\mathrm{e}}$ curves is given (in MW). In column 4, Pci stands for initial power of the CMs, i.e., the power for which the stability margin of column 2 was computed at the iteration of concern; in column 5, $\Delta \mathrm{Pc}$ stands for the suggested change in Pci. In column 6, Pcf stands for the final value of Pc; this is used as the initial Pc value for the next iteration (provided that the critical group does not change from one iteration to the other).

The stabilization procedure starts (iteration $\mathrm{Nr} 0$ ) with the output data of the second block of the FILTRA technique reported in Table II: $\eta_{2}$ (or "minimum distance"), number of CMs, and $\mathrm{Pc}$.

Let us comment on the case $\mathrm{Nr} 13$ of the $\mathrm{H}-\mathrm{Q}$ system, where the group of CMs is the same for all successive simulations.
A first iteration is run using $\Delta \mathrm{Pc}=-(5572-5572 / 1.1)=$ $-507 \mathrm{MW}$. This $\Delta \mathrm{Pc}$ decrease is distributed among $\mathrm{CMs}$, and an increase of the same amount is distributed among noncritical machines. A load flow is then run, followed by a transient stability simulation using SIME. The results are shown in the table: the procedure converges after three iterations; the power of the group of CMs guaranteeing stabilization is finally found to be of 4791 MW (in bold in the table); in other words, stabilizing this case implies a decrease of $14 \%$ of the critical machines' generation power.

The same procedure yields the power limits for the other cases in Table III, as well. Observe that, generally, cases which involve changes of CMs during the procedure and/or very unstable behavior (without initial margin) require a larger number of simulations; nevertheless, this number remains reasonably small (see contingency $\mathrm{Nr} 1$ of the EPRI system which accumulates the two "difficulties").

Many other interesting aspects of the table would deserve further discussion, but are skipped for space reasons.

\section{CONCLUSION}

This paper has proposed a general approach to contingency filtering, ranking and assessment (FILTRA) in transient stability studies. It is made up of two blocks, one for screening contingencies, the other for ranking and assessing the "potentially interesting" ones. Both blocks rely on SIME, a hybrid transient stability method, which achieves fast computation of stability margins and identification of critical machines.

Conceptually, the approach is unified, accurate, flexible and powerful: unified, since it uses the same transient stability package throughout and takes multiple advantages of each computed margin; accurate, since its design achieves a faithful assessment of the time-domain program; flexible, since it may handle any power system modeling, contingency scenario and mode of (in)stability; powerful, since it is able to devise efficient filtering, ranking, assessment and control tools.

From this general two-block structure a particular FILTRA technique has then been considered, complying with the specifics of two power systems, and scrutinized on these systems. Thus, over 600 contingencies have been screened; of them, about $82 \%$ were readily discarded by the filtering block, while the others were classified into harmless (7\%), potentially dangerous $(6 \%)$, and dangerous $(5 \%)$. These latter contingencies were further ranked in terms of severity and assessed in terms of their margin and critical machines. Finally, the control possibilities of the SIME method were tested on a sample of dangerous contingencies, using an automatic iterative procedure. Throughout, the power systems were simulated with detailed modeling. The technique was found to be reliable (i.e., to capture without exception all dangerous contingencies), and to combine accuracy with efficiency. Indeed, it achieves computing performances compatible with real-time requirements, while using detailed power system modeling.

The paper finishes up with an opening to power system control and, in Appendices A and B, with suggestions for more sophisticated designs of the filtering block. 
APPENDIX A

A GlANCE AT SIME

\section{A. Foundations}

The multi-machine power system parameters provided by a time-domain program are transformed into those of a one-machine infinite bus (OMIB) system, and refreshed at each time step of the program. Further, at each time step, the stability of the OMIB is explored by the Equal Area Criterion (EAC); the procedure is stopped as soon as the (in)stability conditions of the EAC are reached (see below).

More precisely, after a contingency inception and its clearance, SIME drives a time-domain program so as to accomplish the following tasks: identify the critical and non critical machines and aggregate them into two groups; replace these groups by successively a two-machine, then an OMIB equivalent system; assess transient stability of this OMIB, using the EAC [1], [2]. The various steps of the method are briefly described below and illustrated in figures corresponding to real stability cases. For more details about SIME, see [16].

\section{B. Identification of the Critical Machines (CMs)}

By definition, the critical machines are those which cause the system loss of synchronism. To identify them, SIME drives the time domain (T-D) transient stability program first in the during-fault, then in the post-fault configuration. And, as soon as the system enters the post-fault phase, SIME starts considering a few candidate decomposition patterns, until one of them reaches the instability conditions (A.3) defined below.

More precisely, at each time step of the post fault simulation, SIME sorts the machines according to their rotor angles, identifies the very first largest rotor angular deviations ("distances") between adjacent machines, and considers as candidate CMs those which are above each one of the (say, 5) largest distances. The procedure is carried out until a candidate group of CMs and corresponding OMIB reaches the unstable conditions (A.3). It is then declared to be the critical OMIB of concern or simply the OMIB. 4

\section{OMIB Parameters, Stability Margins and By-Products}

The OMIB parameters $\delta, \omega, M, P_{\mathrm{m}}, P_{\mathrm{e}}$ are computed from the corresponding individual machines parameters, using the concept of partial center of angle [1], [2] [see in Fig. 3 the OMIB trajectory plotted from the multi-machine trajectories (swing curves)]. On the other hand, the EAC states that the stability margin is the excess of the decelerating area over the accelerating area [see Fig. 3(a)]. Accordingly, the following

\footnotetext{
${ }^{4}$ Note that the above criterion for identifying the CMs and corresponding OMIB obeys the necessary and sufficient conditions derived from EAC and expressed by (A.3). This unambiguous identification of the CMs is a major advantage of hybrid one-machine equivalent methods over hybrid multi-machine methods. Besides, the criterion is free from any pragmatic consideration, unlike T-D methods which call upon pragmatic criteria to detect instability. Finally, the procedure is computationally very unexpensive: it requires computation of candidate OMIB parameters (which is straightforward) and, in addition, it allows saving number and duration of T-D simulations (condition (A.3) is generally reached much earlier than instability conditions used in multi-machine T-D simulations).
}

analytical expressions for unstable and stable margins are derived [1], [2]:

$$
\begin{gathered}
\eta_{\mathbf{u}}=-\frac{1}{2} \mathrm{M} \omega_{\mathbf{u}}^{2} \\
\eta_{\mathrm{st}}=\int_{\delta_{\mathrm{r}}}^{\delta_{\mathrm{u}}}\left|P_{\mathrm{a}}\right| d \delta .
\end{gathered}
$$

In these expressions,

- the accelerating power $\mathrm{P}_{\mathrm{a}}$ is the difference $\mathrm{P}_{\mathrm{a}}=\mathrm{P}_{\mathrm{m}}$ $\mathrm{P}_{\mathrm{e}}$;

- subscript "u" (for unstable) refers to the angle $\delta_{\mathbf{u}}$, speed $\omega_{\mathbf{u}}$, and time $t_{\mathbf{u}}$ when the OMIB instability conditions are met:

$$
\mathrm{P}_{\mathrm{a}}=0 ; \dot{\mathrm{P}}_{\mathrm{a}}>0
$$

- $\quad$ subscript "r" (for return) refers to the angle $\delta_{\mathrm{r}}$ and time $\mathrm{t}_{\mathrm{r}}$ where $\delta$ starts decreasing and $\omega$ vanishes (OMIB stability conditions):

$$
\omega=0 ; \dot{\mathrm{P}}_{\mathrm{a}}<0
$$

Fig. 3 illustrates the EAC in an unstable and a stable cases.

Remarks:

1) The above descriptions show that the computing effort necessary to get an unstable margin is $t_{\mathbf{u}}$ sTDI; similarly, $t_{\mathrm{r}} \mathrm{sTDI}$ is the computing effort for getting a first-swing stable margin. ${ }^{5}$

2) A two-margin linear extra- (inter-)polation provides an approximate value of a contingency critical clearing time (CCT). Such procedures have been sketched in (II), (III) of Fig. 2 and used in Section III.

\section{APPENDIX B \\ DESIGNING ELEMENTARY FILTERS}

The ultimate objective of the filtering block of Fig. 1 is to realize a good compromise between reliability (ability to capture all the dangerous contingencies), efficacy (as low as possible rate of false alarms) and computational efficiency. Note that accuracy is not the main concern at this stage. Hence, many approximate filtering schemes may be thought of, as described below.

A first question of concern is whether and to which extent power system simplified modeling (SM) could be exploited. Actually, this raises the twofold question: i) is the SM at all usable? ii) if yes, does SM give a reasonable account of system behavior, i.e., of the system modeled in its normal (detailed) way? This twofold question may receive many answers:

- "no": the real system modeling is so sophisticated that SM is meaningless;

- "yes," but: SM does give a picture of the real power system behavior but also introduces distortions (e.g., multi-swing

\footnotetext{
${ }^{5}$ But exploration of multi-swing instabilities requires examination of the simulation on the entire maximum integration period.
} 
phenomena which may disappear with the detailed modeling (DM);

- "yes, indeed": the power system behaves in a similar way with SM and DM, though its transient stability limits (power limits or critical clearing times) are generally lower with SM than with DM.

Obviously, systems belonging to the third class are good candidates for a pre-filter with SM. The "yes, but" category is more difficult to apprehend and needs off-line tuning of the considered power system. For example, one should determine whether multi-swing phenomena may exist.

The above considerations lead to three types of filters, using three ways to screen contingencies: i) first-swing (in)stability; ii) approximate CCTs relying on a single-margin; iii) approximate CCTs relying on two margins.

Filter i) has already been described in Section II-C, Fig. 2. Filter ii) computes contingency CCTs using a compensation scheme proposed in [14] and exploited in [15]. Filter iii) may be designed so as to detect multi-swing phenomena; in this case, to save CPU, it is advised to use it only when simplified modeling is practicable (e.g., see [1], [16]).

\section{ACKNOWLEDGMENT}

The authors would like to thank the Electric Power Research Institute (project WO\#3103-10) for developing the filtering part of the FILTRA scheme and for performing investigations on the EPRI system.

\section{REFERENCES}

[1] Y. Zhang, L. Wehenkel, P. Rousseaux, and M. Pavella, "SIME: A hybrid approach to fast transient stability assessment and contingency selection," Int. Journal of Electrical Power and Energy Systems, vol. 19, no. 3, pp. 195-208, 1997.

[2] Y. Zhang, L. Wehenkel, and M. Pavella, "SIME: A comprehensive approach to fast transient stability assessment," Trans. of IEE Japan, vol. 118-B, no. 2, pp. 127-132, 1998.

[3] Y. Xue, Th. Van Cutsem, and M. Ribbens-Pavella, "A simple, direct method for fast transient stability assessment of large power systems," IEEE Trans. Power Systems, vol. 3, no. 2, pp. 400-412, May 1988.

[4] M. Pavella, "Generalized one-machine equivalents in transient stability studies," PES Letters, IEEE Power Engineering Review, vol. 18, no. 1, pp. 50-52, Jan. 1998.

[5] "Standard test cases for dynamic security assessment," Electric Power Research Institute, Project 3103-02-03, Final EPRI report no. EPRI TR-105 885, Dec. 1995

[6] CIGRE Task Force 38.02.17, "Advanced angle stability controls," Carson Taylor, Convener, CE/SC 380217 2000. p. 210.

[7] A. A. Fouad and V. Vittal, "The transient energy function method," Int. Journal of Electrical Power and Energy Systems, vol. 10, pp. 233-246, 1988.

[8] M. A. Pai, Energy Function Analysis for Power System Stability: Kluwer Academic, 1989.

[9] M. Pavella and P. B. Murthy, Transient Stability of Power Systems. Theory and Practice: J. Wiley, 1994.
[10] G. C. Ejebe, C. Jing, J. G. Waight, V. Vittal, G. Pieper, F. Jamshidian, D. Sobajic, and P. Hirsch, "On-line dynamic security assessment: Transient energy based screening and monitoring for stability limits," in 1997 IEEE Summer Meeting, Panel Session on "Techniques for Stability Limit Search".

[11] "Extended transient midterm stability program version 3.1 user's manual," Electric Power Research Institute, Projects 1208-11-12-13, Final EPRI report no. EPRI TR-102 004, May 1994.

[12] A. Valette, F. Lafrance, S. Lefebvre, and L. Radakovitz, "ST600 programme de stabilité: Manuel d'utilization version 701," Hydro-Québec, Vice-Président Technologie et IREQ, 1987.

[13] D. Ruiz-Vega, D. Ernst, C. Machado Ferreira, M. Pavella, P. Hirsch, and D. Sobajic, "A contingency filtering, ranking and assessment technique for on-line transient stability studies," in International Conference on Electric Utility Deregulation and Restructuring, and Power Technologies DRPT2000, London, UK, Apr. 4-7, 2000, pp. 459-464.

[14] D. Ruiz-Vega, A. L. Bettiol, D. Ernst, L. Wehenkel, and M. Pavella, "Transient stability-constrained generation rescheduling," in Bulk Power System Dynamics and Control IV-Restructuring, Santorini, Greece, Aug. 1998, pp. 105-115.

[15] A. L. Bettiol, L. Wehenkel, and M. Pavella, "Transient stability-constrained maximum allowable transfer," IEEE Trans. Power Systems, vol. 14, no. 2, pp. 654-659, May 1999

[16] M. Pavella, D. Ernst, and D. Ruiz-Vega, Transient Stability of Power Systems: a Unified Approach to Assessment and Control: Kluwer Academic Publishers, Sept. 2000.

Damien Ernst is currently a Ph.D. student at the University of Liège (Belgium), and an FNRS Research Associate. His research concerns real-time transient stability emergency control. He is an IEEE Student Member.

Daniel Ruiz-Vega is presently a Ph.D. student at the University of Liège (Belgium). His research concerns real-time preventive transient stability control. He is an IEEE Student Member.

Mania Pavella has been working in the field of electric power system analysis and control. She is an IEEE Fellow.

Peter M. Hirsch is Manager of Software Quality of the Energy Delivery and Utilization Division, at EPRI, and responsible for implementing the EPRI software standards and coordinating software quality within Energy Delivery and Retail Sectors. He is also a project manager in Grid Operation and Planning Department. He received the B.S., M.S. and Ph.D. degrees from the University of Wisconsin. Dr. Hirsch is a Senior Member of the IEEE.

Dejan Sobajic is presently Manager of Grid Operation and Planning at the Electric Power Research Institute in Palo Alto, CA. He is also a consulting Associate Professor with Electrical Engineering Department at Stanford University. He is a Senior Member of the IEEE. 